\title{
L'argumentation à la Cour d'Assises brésilienne : les émotions dans le genre du rapport de police
}

Argumentation at the Brazilian criminal court: emotions and power relations in the police report

Helcira Maria Rodrigues de Lima

(2) OpenEdition

Journals

Édition électronique

URL : https://journals.openedition.org/aad/1155

DOI : 10.4000/aad. 1155

ISSN : 1565-8961

Éditeur

Université de Tel-Aviv

Référence électronique

Helcira Maria Rodrigues de Lima, «L'argumentation à la Cour d'Assises brésilienne : les émotions dans le genre du rapport de police ", Argumentation et Analyse du Discours [En ligne], 7| 2011, mis en ligne le 15 octobre 2011, consulté le 21 septembre 2021. URL : http://journals.openedition.org/aad/1155 ; DOI : https://doi.org/10.4000/aad.1155

Ce document a été généré automatiquement le 21 septembre 2021.

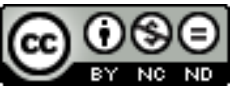

Argumentation \& analyse du discours est mis à disposition selon les termes de la licence Creative Commons Attribution - Pas d'Utilisation Commerciale - Pas de Modification 4.0 International. 


\section{L'argumentation à la Cour d'Assises brésilienne : les émotions dans le genre du rapport de police}

Argumentation at the Brazilian criminal court: emotions and power relations in the police report

Helcira Maria Rodrigues de Lima

\section{Introduction}

Personne ne jouit d'une liberté totale : nous commençons opprimés par la syntaxe et finissons aux prises avec le Commissariat au maintien de l'ordre politique et social, mais dans les étroites limites que nous laissent la grammaire et la loi nous pouvons encore bouger. Graciliano Ramos

1 Cet article se propose d'engager une réflexion ${ }^{1}$ sur le rôle que joue l'émotion dans la construction du discours de la Cour d'Assises au Brésil, en tenant compte des relations de pouvoir qu'elle instaure. Il se penche plus particuliement sur un genre qui est propre à cette instance judiciaire, le Boletim de Ocorrências (BO), ou premier rapport écrit par le commissaire de police. Il ne s'agit bien sûr que d'un genre de discours parmi tous ceux dont relèvent les pièces versées à un procès criminel : s'y ajoutent, entre autres, les dépositions de témoins et de l'accusé, l'arrêt de mise en accusation, les rapports des experts. Le choix du BO a cependant l'avantage de montrer la difficulté à concevoir un texte totalement dépourvu de marques d'émotion, ou qui ne suscite aucun sentiment dans l'auditoire: il s'agit en effet (ou il devrait s'agir) d'une présentation extrêmement technique et de ce fait rédigée de manière neutre, ainsi que le préconisent les manuels de la police judiciaire ${ }^{2}$. On peut ainsi voir comment, sous 
l'apparence d'une neutralité calculée, l'émotion constitue en fait un élément important de la production argumentative du discours de la Cour d'Assises.

2 Je prendrai à titre d'exemple un Boletim de Ocorrências (BO) rédigé après l'assassinat d'une femme qui a eu lieu en 1980, à Belo Horizonte (Brésil). Il fait partie des pièces d'un procès criminel déjà archivé, ce qui m'a permis d'y avoir accès. Le crime en question a choqué à l'époque l'opinion publique pour avoir relancé la discussion sur la violence exercée contre les femmes, ainsi que sur les valeurs conservatrices et machistes sur lesquelles se fondait (et en réalité se fonde toujours) la société brésilienne et plus particulièrement la société "mineira» (adjectif qualifiant les habitants de l'Etat du « Minas Gerais » - au Brésil : mineiro[a]). L'importance accordée à ce crime, devenu emblématique, est due non seulement à la position sociale de l'accusé (A) et de la victime $(\mathrm{V})^{3}$ ( $\mathrm{V}$ était une riche femme d'affaires et $\mathrm{A}$ un ingénieur de renom), mais aussi aux débats qu'il a suscités, ravivant les discussions qui s'étaient donné libre cours lors de l'assassinat de deux autres «mineiras » : Jô de Lima e Souza et Ângela Diniz ${ }^{4}$. Aujourd'hui encore, à l'occasion de procès criminels traitant de crimes dits " passionnels », la mort de ces trois jeunes femmes est rappelée.

Cet article se divise en trois parties, outre l'introduction et les considérations finales. La première présente la conception de l'argumentation qui sous-tend l'analyse, et qui se fonde sur la contribution de la rhétorique classique et de l'argumentation dans la langue. La seconde partie consiste en une brève lecture de la structure et du fonctionnement de la Cour d'Assises et en une présentation, dans ce cadre, du genre du BO. Elle se propose de mettre en évidence les éléments qui permettent d'éclairer cet organisme de la Justice criminelle en général, et de mieux saisir le rôle du BO au moment du jugement, en particulier. Enfin, dans la troisième partie, j'analyserai brièvement le texte du BO sélectionné pour en montrer le fonctionnement et en dégager la dimension argumentative.

\section{Une rapide mise au point sur l'argumentation}

4 L'argumentation présuppose l'adhésion intellectuelle de quelqu'un qui se mobilise en vue d'une action déterminée. Cette action peut appeler à une réponse immédiate ou seulement à un changement d'attitude auprès de l'auditoire. L'argumentateur doit connaître l'allocutaire qu'il désire persuader et miser sur ses goûts, ses croyances, ses connaissances ; il lui faut définir le profil de cet autre afin d'élaborer une stratégie qui puisse l'atteindre.

Dans ce cadre, nous reprenons ici la grande tradition rhétorique qui confère à l'argumentation sa triple dimension: la dimension pathémique, la dimension de la construction des images de soi et de l'autre, et la dimension démonstrative ${ }^{5}$. Cette division, qui reprend la triade aristotélicienne, permet de pousser plus loin l'analyse du discours de la Cour d'Assises dans la mesure où elle donne à comprendre l'argumentation qui s'y déroule, non seulement comme liée à une certaine rationalité, mais aussi comme offrant un tout constitué par les trois éléments dégagés par le philosophe : l'ethos, le pathos et le logos.

6 La première dimension, pathémique, est liée à la mobilisation des émotions dans un but persuasif : il s'agit d'émotions mues par une visée. Sans doute l'analyste ne dispose-t-il pas d'instruments lui permettant d'évaluer quelle émotion est effectivement ressentie 
par le locuteur : c'est à une lecture des éléments verbaux susceptibles de susciter des émotions chez l'allocutaire qu'il se livre. Toutefois, s'il est difficile d'évaluer dans quelle mesure l'émotion exprimée est réellement ressentie, il faut préciser que sur la base des travaux de Wierzbicka (1999), Kerbrat-Orecchioni (2000) ou Plantin (1997, 2003), il paraît possible de dégager les traces discursives des émotions dans le tissu verbal.

Quant à la dimension de la construction des images (de soi et de l'autre), elle est liée à la notion d'ethos rhétorique bien que notre modèle ne se restreigne pas à l'image de soi dans le discours. L'autre - et l'image qui en est offerte - n'est pas seulement présent en sa qualité de destinataire idéal, mais aussi et surtout en tant qu'instance construite par le discours. Au moyen de cette construction qui s'effectue dans l'énonciation, il est possible au locuteur de mieux ériger sa propre image de façon à s'octroyer la légitimité et la crédibilité nécessaires à son entreprise de persuasion.

La troisième et dernière dimension, appelée démonstrative, se réfère à un usage du langage qui se fonde sur une rationalité qui, si elle n'est absente des autres dimensions, est ici plus calculée. Elle s'appuie non seulement sur des schémas de raisonnement et une organisation du discours qui se targuent d'emporter la conviction, mais aussi sur l'utilisation de preuves techniques comme les comptes rendus, les documents, les photographies, etc.

9 Bien qu'elles soient presque toujours présentes d'une façon ou d'une autre dans le discours, ces trois dimensions peuvent interagir en conférant une position plus ou moins dominante à l'une d'entre elles. La métaphore des plateaux, développée par Deleuze (1998), permet de réfléchir à cette interrelation constitutive de l'argumentation. Se référant à l'organisation de son livre « Mille Plateaux » dans une interview accordée au quotidien Libération, le philosophe parle de l'image des "plateaux" qu'il considère comme un ensemble d'anneaux rompus qui peuvent s'interpénétrer : «Chaque anneau ou chaque plateau devrait avoir son propre climat, son propre timbre» (Deleuze 1998: 37). Ce mélange de fermeture et d'ouverture de chaque plateau, cette singularité en même temps que cette interpénétration mutuelle reflètent bien les relations qui caractérisent les trois dimensions du discours argumentatif. Comme les plateaux, chaque dimension possède sa propre vie, mais comme une voie d'accès aux autres. Elles peuvent aussi bien s'unir en un processus d'intercession formant un tout, ayant un sens, que se séparer en manifestant leur indépendance. En bref, les trois dimensions sont liées; elles sont simultanément dépendantes et indépendantes l'une de l'autre.

10 Au-delà de cette réélaboration de la tradition rhétorique, il faut tenir compte du fait que l'argumentation traverse, voire constitue le discours, si bien que son analyse doit tenir compte du « dispositif de l'énonciation et de la dynamique interactionnelle, sans laisser de côté les données institutionnelles, sociales et historiques » (Amossy $2000: 7$ ). Pour comprendre une telle dynamique, il est nécessaire d'étudier attentivement les conditions de la production du discours, ce qui permet à la fois de penser l'argumentation en liaison avec les relations de pouvoir qui vont au-delà des interactions, et de se recentrer sur la matérialité linguistico-discursive. C'est en effet dans cette matérialité que l'on peut saisir les marques laissées par l'énonciation et c'est sur elle que l'on pourra appuyer nos analyses. Pour ce faire, l'étude de l'argumentation doit se fonder aussi bien sur la rhétorique classique que sur les travaux de sémantique argumentative dits "argumentation dans la langue». Dans cette perspective, et contrairement à ce que préconise Ducrot (2004), il nous semble qu'une analyse 
argumentative se doit d'associer l'«argumentation rhétorique " à l'«argumentation linguistique $»^{6}$.

\section{La Cour d'Assises et ses acteurs}

11 L'architecture actuelle de domination de la Cour d'Assises brésilienne impose un certain régime de parole et de visibilité. Des mots et de la lumière : ce qui peut se dire et ce qui peut se voir. La structure matérielle du tribunal représente bien l'architecture du pouvoir: la place de chacun y est préétablie et différenciée d'un point de vue physique et symbolique ${ }^{7}$. Au tribunal, et au cours du procès, chacun occupe une place déterminée en fonction de sa position dans la hiérarchie sociale. Et en effet, la structure spatiale du tribunal représente bien l'ordre social en vigueur : à la droite du Juge se tient le Procureuret à sa gauche la défense. Plus loin se tiennent les Jurés et plus loin encore l'Accusés. Les différents paliers de la structure spatiale sont un indice de la hiérarchie sociale. Ainsi, la place de chacun dans la société est-elle reproduite au tribunal.

En ce qui concerne les pièces du procès, d'une manière générale, il y a plusieurs "acteurs juridiques ${ }^{9}$ impliqués dans leur construction en plus des témoins et de l'accusé. Le texte procédural met en scène la voix non seulement d'un, mais de plusieurs individus, sous la houlette du juge. Le policier est le premier à se manifester lors du procès, car le BO suit immédiatement la dénonciation du crime.

Dans la mesure où le «Boletim de Ocorrências " tel qu'il est entendu au Brésil, et surtout dans le cas de ce travail, n'existe pas en France, où c'est le commissaire (ou éventuellement un inspecteur) qui rédige le premier rapport écrit, quelques mots d'explication sur ce genre judiciaire s'imposent ${ }^{10}$. De quoi s'agit-il exactement? Après la dénonciation du crime par la victime, ou un tiers (en cas de tentative d'homicide), à l'autorité policière, un employé (en général un greffier de la police ou un simple policier) est désigné pour aller évaluer la situation sur les lieux du crime et rédiger un rapport - le BO. Ce texte devra présenter, de manière technique et succincte, des informations concernant la victime, l'agresseur, le lieu et l'heure du crime et les données sur les premières mesures prises pour l'enlèvement du corps, l'expertise sur place, etc. ${ }^{11}$ C'est à partir du BO que l'affaire entre dans le cadre du droit. C'est pourquoi le réseau de signes qui l'entoure et qui en découle est l'une des phases les plus importantes de l'enquête policière, car ce document joue un rôle fondamental dans la direction que suivra celle-ci et constitue l'élément principal qui permet de découvrir l'auteur du crime. Si le BO apparaît comme un texte très court en regard de la gravité de la situation, il n'en joue pas moins le rôle du passeport qui permet de pénétrer dans un monde très particulier.

Après le BO viennent les pièces relatives au résultat de l'enquête menée par le commissaire: interrogatoire des témoins, de l'accusé, examens médicaux, photographies des lieux du crime et de la victime, photographies des objets appartenant à la victime et/ou à l'accusé. Certains objets comme une plaquette de comprimés ou une mèche de cheveux peuvent être annexés au procès. Toutes ces procédures visent à faire surgir des indices de la culpabilité de l'accusé et de la «matérialité » du crime. La version juridique commence à être construite à partir de chaque geste, de chaque mot, et du comportement des individus. Elle est traduite en articles et qualificatifs aggravants et atténuants. D'un autre côté, comme l'affirme 
Izumino (1998: 65), «toute la vie des personnes impliquées est exposée : leurs drames particuliers, leurs secrets les mieux gardés, leurs vices et leurs vertus ». Tout ce travail est réalisé au moyen d'éléments et d'informations apparemment simples.

Lorsque l'enquête policière est terminée, tout ce qui la concerne est envoyé à la justice par le commissaire avec un rapport final qui doit présenter son opinion sur le crime et les éléments recueillis jusque-là. Il faut noter que ce moment constitue une rupture, une limite qui doit être observée avec prudence. En effet, cette appréciation du commissaire donne de l'affaire une image qui est certainement affectée par une vision du monde spécifique et qui peut dès lors apposer sa marque sur la manière dont sera jugé le crime. Malgré la distance qui le sépare spatialement de la salle de la Cour d'Assises, la voix du commissaire y sera présente et, s'appuyant sur celle du policier, des experts, des témoins et de l'accusé, elle condamnera celui-ci ou l'absoudra. Ses valeurs, ses croyances, ses désirs et ses connaissances se répercuteront d'une manière ou d'une autre sur la sentence finale. Toutefois, si la voix du commissaire possède une telle importance, la voix des autres acteurs n'est, à mon avis, pas moins importante : tous interfèrent dans le cours du procès.

16 Après l'intervention de policiers, d'experts, de témoins et du commissaire, entre en scène la personne du procureur, représentant du Ministère public; ensuite le juge se manifeste. D'autres acteurs juridiques peuvent entrer et sortir de scène suivant l'évolution du procès. Il est intéressant de noter qu'à ce moment les pièces du procès commencent à vivre leur propre vie et, comme l'affirme Mariza Correa (1983:40) :

$\mathrm{Au}$ moment où les actes se transforment en actes écrits, les faits en versions, le fait concret perd presque toute son importance et le débat a lieu entre acteurs juridiques, chacun utilisant la portion du réel qui renforce le mieux son point de vue. Dans ce sens, c'est le réel qui est en procès, un réel pressé jusqu'à ce que l'on puisse en extraire un plan élémentaire à partir duquel se construira un modèle de culpabilité et un modèle d'innocence.

17 Autrement dit, le BO, les rapports techniques des experts, les objets annexés au procès, les photographies, le rapport du commissaire, l'acte d'accusation, le réquisitoire, les interventions du juge et d'autres acteurs juridiques et tout ce qui se trouve faire partie de ce grand texte que constituent les pièces du dossier, concourent tous à la production du discours de la Cour d'Assises. Et bien sûr, à chacun d'eux incombe une certaine responsabilité dans la mesure où il véhicule des croyances et des valeurs qui ont un impact sur le jugement final, et suscite des émotions qui ne manquent pas de l'influencer.

On peut donc affirmer que les pièces du procès constituent l'instance qui interagit le plus fortement avec les membres du tribunal, étant donné que tout le monde y a un accès direct - dans le cas des parties intéressées et des avocats - ou indirect - pour les jurés et le public qui assiste au jugement. Pendant le procès, un dialogue permanent s'instaure entre tous les participants de l'affaire, mais aussi séparément avec chaque pièce du procès. Les divers acteurs en ont conscience lorsqu'ils construisent leurs interventions à l'intention de leurs éventuels interlocuteurs.

19 Ainsi le policier, qui a la responsabilité d'être présent sur les lieux du crime lorsque la police est appelée, rédige le $\mathrm{BO}$ en pensant à un individu spécifique, le commissaire, tout en sachant que ce texte pourra être lu et utilisé par d'autres personnes durant le procès et même par les jurés, de manière directe ou indirecte, au moment du jugement. Les experts et les médecins légistes s'adressent également au commissaire de police et 
celui-ci s'adresse au juge au moyen d'un rapport contenant toutes les données relatives au cheminement de l'investigation du crime. La construction du procès se fait alors par l'entrecroisement de toutes ces voix et d'autres encore qui, porteuses d'intentionnalité, s'organisent autour de visées (Charaudeau 2001) à l'origine de leurs stratégies propres. Dans l'espace de la Justice pénale, chacun se définit en fonction de sa position dans un ordre hiérarchique, lui-même déterminé a priori par des facteurs sociaux et culturels. De cette façon, la force qu'acquièrent les pièces du dossier se manifeste dans un tissu verbal où les relations de pouvoir vont au-delà des discours produits par les acteurs juridiques; ce sont des relations de pouvoir extérieures qui sous-tendent ces discours et leur donnent forme. On voit ici que le pouvoir se multiplie à la manière d'une toile d'araignée, il se dissémine sous le choc discursif déclenché par des individus dont l'objectif est de faire prévaloir leur point de vue, comme s'ils luttaient pour une sorte d'hégémonie argumentative.

Ainsi, à partir du moment où le policier élabore le BO, il met en branle avec d'autres personnes, qui auront accès aux données en circulation, une version du crime. Tous les agents construisent des versions et celles-ci, bien que visant à la neutralité, portent en elles la marque de chacun de leurs producteurs. Quand il s'agit d'un homicide, la situation tend à devenir plus délicate encore, car elle implique des représentations qui peuvent susciter des émotions d'ordres divers. Evidemment, d'autres types de crimes peuvent le faire également, et le font, mais la mort incommode toujours plus. C'est dans ce sens qu'une lecture du rôle de l'émotion lors de jugements en Cour d'Assises devient essentielle pour mieux comprendre tant le fonctionnement de cet organisme, que celui de l'argumentation qui y prend place.

\section{Brève analyse du BO}

21 Dans le BO analysé, des informations importantes relatives aux lieux et aux circonstances du crime ont été notées sur le formulaire standard, selon la structure suivante ${ }^{12}$ :

a. en-tête (numéro du BO ; date ; heure ; nature du crime ; brigade à laquelle appartient le policier, etc.) ;

b. personnes citées (les personnes impliquées dans le crime - victime et accusé, témoins);

c. armes - objets - matériel appréhendés (lorsqu'il y a quelque chose de significatif sur les lieux du crime) ;

d. historique [cette section se réfère à la synthèse réalisée par le policier à partir des informations recueillies (lieux, temps, personnes impliquées, etc.)]. C'est à cet endroit que le policier devra exposer, de manière succincte, claire, impartiale et objective, les circonstances du crime (description des personnes impliquées, heure du crime, description des lieux, description du corps de la victime, s'il y a lieu, etc.).

Les parties se référant à l'en-tête, la personne et l'arme sont remplies de manière quasi automatique et ne dépassent pas ce niveau, mais dans la partie relative à l'historique, la description prend une autre allure. C'est dans cet espace, en effet, que le policier devra présenter des informations plus détaillées sur le crime, fournissant au commissaire des éléments qui n'apparaissent pas dans la partie introductive du document. De plus, ici, l'accent est mis sur l'objectivité, la clarté, la concision et l'impartialité. Cela exige d'éviter l'usage d'adjectifs et d'adverbes, de vocatifs, de lieux communs; de ne pas 
répéter les informations qui figurent déjà dans la partie initiale du document; de ne pas laisser des parties en blanc (tous les espaces doivent être remplis, ou signalés comme s'ils l'étaient, pour éviter les fraudes). Il faut également que l'écriture soit lisible, que le discours indirect prédomine, que le discours direct ne soit utilisé que pour rendre compte de menaces ou d'un manque de respect. Toutes ces instructions sont données aux policiers lors de leur entraînement, mais l'on sait qu'elles ne sont pas toujours respectées.

Dans le cas qui nous concerne, les informations ont été notées de la manière suivante :

Nous sommes allés à l'endroit cité ci-dessus, où nous avons été informés par le premier tém. qu'il a entendu des coups de feu, aux env. de 1 h15 à l'int. de la mais. où il travaille, et aussitôt après, son patron est sorti au portail, prenant une direction inconnue et n'est pas revenu jusqu'à maintenant.

Sur les lieux, j'ai constaté que, la victime gît sans vie, étalée par terre, dans [incompréhensible] de la chambre de sa mais. J'ai donc communiqué à la Copom, et envoyé les Experts $\mathrm{R}$ et $\mathrm{C}$ et $\mathrm{O}$. Je leur ai transmis le fait (la circonstance). Communiqué à vous-même ci-dessus cité ${ }^{13}$.

Comme le $\mathrm{BO}$ est en fait le point de départ de la procédure de l'enquête policière, l'énonciateur semble avoir fait ce qu'il fallait pour répondre aux règles de sa construction : objectivité, concision et technicité. Il existe en effet un contrat qui régit cette construction textuelle et qui implique d'obéir, dans un espace limité, à un modèle. Une telle organisation textuelle répond à des raisons techniques, car il existe un formulaire standard où l'agent doit présenter un certain type d'informations. Il faut dire que les données, en plus de se couler dans un modèle esthétique de construction textuelle, doivent aussi suivre un patron qui astreint le policier à une précision prétendument scientifique.

Ainsi, obéissant au contrat, le policier présente quelques éléments nouveaux par rapport à la description de la première partie : le gardien a entendu des coups de feu à l'intérieur de la maison, aux environs de $1 \mathrm{~h} 15$. Aussitôt après son patron est parti en voiture empruntant une direction inconnue. Il (le policier) a constaté que la victime était sans vie, dans une chambre de la maison. L'arme du crime n'a pas été trouvée et il y a eu cinq ou six coups de feu.

26 L'utilisation de la modalité délocutive (verbes et pronoms à la première personne du pluriel et discours rapporté) par l'énonciateur a pour but de le faire disparaître du texte, en se servant de la "voix» de sa corporation («nous sommes allés», «nous avons été informés par le premier témoin »). A travers cet effacement énonciatif, il permet une énonciation en apparence objective. Grâce à cela, il joue le rôle d'une sorte de porte-parole qui n'accomplit qu'un travail technique, ce qui lui permet d'impliquer l'autre, de le mêler à sa propre voix. Ce n'est pas lui qui a affirmé que l'assassin est parti au milieu de la nuit, mais le gardien de la maison. Ce mode d'énonciation est évident lorsqu'il s'agit de certaines informations qui ne pouvaient être effectivement vérifiées sur les lieux du crime ou qu'il n'avait pas la possibilité de voir lors de son évaluation initiale. On ne peut savoir, par exemple, si le premier témoin (le gardien de la maison) a vraiment entendu les coups de feu ; on ne peut prouver quelle a été l'arme utilisée et si elle appartenait à l'accusé ou à la victime, ni pourquoi cet objet n'était pas à côté du corps de $\mathrm{V}$ ou dans une autre pièce de la maison. Toutes ces données donneront lieu postérieurement à une enquête et à ce porte-parole de la police, il revient seulement de décrire ce qu'il a effectivement vu. 

l'asepsie nécessaire au texte, puisqu'elle met en évidence un réseau de sens lié au discours scientifique et exclut dans un certain sens l'autre - l'interlocuteur. Il s'agit d'une assertion dont la structure se fonde - ou entend se fonder - sur « des savoirs de connaissance » (Charaudeau 2005).

la mesure où l'objectif de l'historique du BO est d'expliquer ce que le policier a vu sur les lieux du crime, le texte tient le rôle qui lui a été imparti. L'énonciateur, tout comme ceux des textes scientifiques, présente une construction objective du monde, au moyen des composantes de la " dénomination », la « localisation » et la " qualification » (Charaudeau 1992). En ce qui concerne la qualification, on perçoit une certaine parcimonie des détails qui auraient pu produire, outre une réalité plus cohérente, une description plus précise de la scène du crime. Les rares informations, bien qu'elles marquent une certaine adéquation, mettent en évidence l'insuffisance des renseignements offerts, ce qui est confirmé par l'extrait du rapport du commissaire de police rédigé après l'enquête :

A, le mari, l'assassin, n'était pas chez lui. Il était parti exactement à 1 h20, tranquillement, en voiture, peu après que tous les gens qui étaient dans la maison (à l'exception des enfants et de la cuisinière $\mathrm{T7}$ ), ont entendu un grand bruit, très vite identifié par le gardien, T5, (fls 05/05) comme étant celui de coups de feu. A ce moment-là, personne dans la maison, ni le gardien, T5, ni la nurse, T6, (fls 12/15), ni la bonne, T8 (qui ne s'est pas réveillée), ne pouvait s'imaginer que la patronne était assassinée par le patron, A. La police n'a été informée de ce qui s'était passé que vers 9 h30 du matin, elle est alors allée sur les lieux où se trouvaient déjà parents et amis de l'assassin [...] (c'est moi qui souligne).

Comme on a pu le constater, il n'est pas fait mention dans le BO de la présence d'amis ni de membres de la famille de l'assassin sur les lieux du crime quand le policier y est arrivé, au contraire de ce qu'indique le rapport du commissaire. Ce dernier fournit de nouvelles informations à partir des dépositions recueillies au commissariat et de l'enquête policière qui a commencé après l'élaboration du BO. Il semble possible, à partir de tels éléments, d'imaginer que le texte analysé n'a pas offert d'informations significatives pour la (re)construction de la scène du crime. La pression exercée par la famille et les amis de l'assassin, personnes influentes de la société mineira de l'époque, a probablement intimidé le policier et explique cette lacune. En effet, on sait qu'il est resté sur les lieux du crime, écoutant les témoins et les autres personnes présentes,

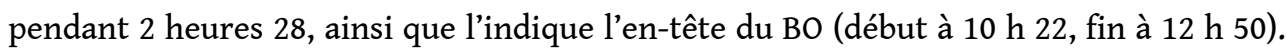
Il est donc clair qu'il a disposé du temps nécessaire pour recueillir des données sur le crime et insérer dans le texte une information aussi importante que celle de la présence des parties intéressées à la défense de l'assassin.

Dans ce sens, si d'un côté l'absence de ces données montre le souci qu'a le locuteur de se protéger et d'assurer la crédibilité de son discours, d'un autre côté, l'absence des informations contenues dans le rapport du commissaire révèle une certaine prise de position de la part du rédacteur du BO. En outre, en utilisant la première personne du singulier pour les verbes "constater » et " communiquer", le locuteur démontre que, d'une certaine manière, il s'implique dans son discours. Qui plus est, le présent historique ( $\mathrm{j}$ 'ai constaté que la victime gît sans vie ») fait surgir la scène du crime sur le papier. C'est comme si par la présentation de ces données, l'individu souhaitait évoquer dans la tête du lecteur l'image du crime: V «étalée par terre dans la chambre ». Dans ce sens, il peut être lu comme un biais émotionnel. En d'autres termes,

Argumentation et Analyse du Discours, 7 | 2011 
bien qu'il ne s'agisse pas d'un "verbe psychologique ", "constater » à la première personne du singulier révèle le trouble provoqué par la présence de la famille et des amis de l'assassin sur la scène du crime. Cela se voit car l'oscillation - première personne du singulier et première personne du pluriel - est révélatrice d'une tension et d'un conflit, étant donné que le locuteur manifeste une prise de distance, mais explicite en même temps qu'il est proche du crime.

31 Le choix du verbe "étaler ", toujours sur le même mode, dont le signifié est "faire tomber ou tomber de tout son long, jeter par terre» (Ferreira 1985: 583), peut également mener à une lecture émotionnelle. Le BO fera partie du rapport qui sera remis par le commissaire au Ministère public et c'est à partir de lui que commencent les investigations. Son contenu sera donc repris plusieurs fois pendant l'enquête. Sur cette base, il semble possible d'affirmer que l'utilisation du terme "étalée " au lieu de "étendue par terre ", par exemple, peut susciter plus d'émotions chez le lecteur du texte. Son sens se rapproche du réseau sémantique d'un autre verbe " jeter (par terre) » qui a acquis, tout comme le premier, un caractère négatif dans notre société et veut dire désormais faire «tomber violemment». C'est dans ce sens que l'on peut affirmer qu'il existe une certaine orientation émotionnelle dans le texte du BO. Il n'est évidemment pas possible de poser que le policier a eu cette intention lors de la rédaction du compte rendu, ni qu'il s'est livré à une sélection lexicale méticuleuse dans le but de déclencher une émotion chez son allocutaire ; mais il a laissé échapper dans son texte, apparemment dépourvu de marques de subjectivité ou de visée émotionnelle, des éléments qui, dans ces circonstances, pourraient laisser percer des traces affectives et des éléments susceptibles de susciter l'émotion. Dans ce cas précis, on peut aussi imaginer que l'individu interprétant puisse être choqué, ou même indigné, par le caractère succinct et « aseptique » du rapport du policier. Ainsi, la relation de la scène du crime apparemment neutre et objective (ou l'absence manifeste de données significatives dans cette même relation) contribue, lorsqu'elle est lue dans sa relation avec les autres parties de l'enquête, à conférer une orientation émotionnelle à la description.

Il faut ici souligner que l'émotion dans le discours n'est jamais le fruit d'une relation mécanique : elle découle d'une construction argumentative des émotions. En d'autres termes, la construction des émotions permet éventuellement de mettre en place certaines relations, mais ne provoque pas de réaction automatique. Je suppose, en suivant Plantin (1997), que de telles relations sont tangibles en raison des scripts que nous avons déjà élaborés en fonction de nos connaissances sur les crimes et tout ce qui les entoure. A partir des considérations de Plantin, on peut penser que les questions «qui ? ", « où ? ", « comment? " et « combien de coups de feu? » auxquelles a répondu le policier, font parties de "questions topiques" d'émotion dans ce texte car elles relèvent les éléments qui orientent vers une possible pathémisation. Il est important de souligner, une fois de plus, que les réponses seront reliées entre elles par l'individu interprétant et, bien que dépendant d'un certain bagage, ces liaisons ne s'effectuent pas mécaniquement. C'est la " couture » de tous les éléments liés à l'enquête et la manière dont ils seront repris par les avocats qui pourra provoquer ou non un certain type d'effet pathémique chez les jurés ${ }^{14}$.

Lorsqu'il quitte la scène, le policier transfère la responsabilité d'une observation plus détaillée de la scène du crime aux experts et délègue au commissaire l'obligation de la coordination des nouvelles procédures. 


\section{Considérations finales} les acteurs juridiques, indépendamment de leur position dans le système hiérarchique, interfèrent d'une certaine manière dans l'affaire. Tous, sous une apparente neutralité scientifique, prennent position, contribuent à l'élaboration du verdict et agissent émotionnellement sur le déroulement du procès car de même que le pouvoir de la Cour d'Assises ne se situe pas chez l'un ou l'autre individu, ou dans l'une ou l'autre instance, les émotions ne sont pas suscitées uniquement par la défense et l'accusation. Si, comme nous l'enseigne Foucault (1999), le pouvoir se dissémine en toile d'araignée, les émotions elles aussi se dispersent et se disséminent dans les discours des différents locuteurs, et jusque dans ceux qui se donnent comme les plus factuels. 


\section{BIBLIOGRAPHIE}

Amossy, Ruth (éd.). 1999. Images de soi dans le discours. La construction de l'ethos (Lausanne : Delachaux et Niestlé)

Amossy, Ruth. 2000. L'argumentation dans le discours. Discours politique, littérature d'idées, fiction (Paris : Nathan)

Charaudeau, Patrick. 1992. Grammaire du sens et de l'expression. (Paris : Hachette)

Charaudeau, Patrick. 2001. « Visées discursives, genres situationnels et construction textuelle », Ballabriga, Michel (éd.), Analyse des discours. Types et genre, communication et interprétation (Toulouse : Éditions Universitaires du Sud), [En ligne : http://www.patrick-charaudeau.com/ Visees-discursives-genres,83.html, consulté le 20 juillet 2011]

Charaudeau, Patrick. 2004. «Comment le langage se noue à l'action dans un modèle sociocommunicationnel du discours. De l'action au pouvoir », Cahiers de Linguistique Française 26, 151-175

Charaudeau, Patrick. 2005. Le discours politique : les masques du pouvoir (Paris : Vuibert)

Correa, Mariza. 1983. Morte em família: representações jurídicas de papeis sexuais (Rio de Janeiro : Rocco)

Deleuze, Gilles. 1998. Conversações (São Paulo: Editora 34)

Doury, Marianne. 2000. «La réfutation par l'accusation d'émotion : exploitation argumentative de l'émotion dans une controverse à thème scientifique ", Plantin, Christian, Marianne Doury \& Véronique Traverso (éds.), Les émotions dans les interactions (Lyon : Presses Universitaires de Lyon), 265-277

Ducrot, Oswald. 2004. « Argumentation rhétorique et argumentation linguistique », L'argumentation aujourd'hui. Positions théoriques en confrontation (Paris : Presses Sorbonne Nouvelle) Ferreira, Aurélio Buarque de Holanda. 1985. Novo dicionário Aurélio (Rio de Janeiro : Nova Fronteira)

Foucault, Michel. 1999. Microfísica do poder. Organização e tradução de Roberto Machado. (Rio de Janeiro : Graal)

Izumino, Wânia Pasinato. 1998. Justiça e violência contra a mulher - o papel do sistema judiciário na solução dos conflitos de gênero (São Paulo : Anablume)

Kerbrat-Orecchioni, Catherine. 2000. «Quelle place pour les émotions dans la linguistique du XXe siècle? Remarques et aperçus ", Plantin, Christian, Marianne Doury \& Véronique Traverso (éds.), Les émotions dans les interactions (Lyon : Presses Universitaires de Lyon), 33-74

Lima, Helcira Maria Rodrigues de. 2006. Na tessitura do Processo Penal: a argumentação no Tribunal do Júri. Tese (Doutorado em Linguística, Faculdade de Letras, UFMG, Belo Horizonte)

Lima, Helcira Maria Rodrigues de. 2007. «L'arène des pas perdus : une relecture de la Cour d'assises brésilienne », Le Brésil à l'épreuve de la modernité. Discours, savoir et pouvoir (Paris : L'Harmattan)

Lima, Helcira Maria Rodrigues de. 2011 (sous presse). « Emoções, racionalidade e diferença » (São João Del Rey : UFSJ). Anais do IV Encontro Mineiro de Análise do Discurso : interfaces da Análise do Discurso em Minas Gerais 
Plantin, Christian. 1996. «L'argumentation dans l'émotion », Pratiques 96, 81-100

Plantin, Christian. 2003. «Structures verbales de l'émotion parlée et de la parole émue », Colleta, Jean-Marc \& Anna Tcherkassof (éds), Les émotions : cognition, langage et développement (Bruxelles : Mardaga), 97-130

Ramos, Graciliano. 1994. Memórias do cárcere (São Paulo : Record)

Wierzbicka, Anna. 1999. « Emotional Universals », Language Design 2, 23-69

\section{NOTES}

1. Tirée d'une étude plus vaste réalisée dans le cadre d'une thèse de doctorat

2. Comme dans ce cas il s'agit d'un crime qui a été commis en 1980, le BO a été rédigé selon les règles en vigueur à l'époque. Des changements ont eu lieu depuis afin de rendre plus claires et moins ambiguës les informations contenues dans ce document. Nous ne les analyserons pas en détail ici.

3. L'utilisation des lettres "V" pour victime et "A" pour accusé et de "T" pour les témoins vise à protéger les personnes concernées par le crime.

4. Jô de Lima e Souza qui a été assassinée par son mari à la fin des années 60 et Ângela Diniz qui a été tuée par son compagnon dans les années 70, faisaient parties de l'élite sociale de l'époque. C'est peut-être pour cela que ces crimes ont suscité de nombreux débats sur la violence contre les femmes au Brésil, en particulier auprès des féministes et des sympathisantes de la lutte pour la condamnation des assassins et la suppression de l'argument de la " légitime défense de l'honneur et de la dignité » qui figure dans le Code pénal brésilien. Il faut néanmoins rappeler que, malheureusement, tous les jours, des femmes sont assassinées. Mais la mort d'un membre d'une classe sociale élevée attire plus l'attention, en montrant que ce type de crime n'est pas l'apanage des pauvres.

5. Pour plus d'information sur le sujet cf. Lima (2006).

6. Ducrot (2004) expose les raisons qui l'ont amené à s'éloigner de l'argumentation rhétorique et "à défendre une argumentation linguistique ».

7. Les rapports de pouvoir à la Cours d'Assises sont explicités dans Lima (2006).

8. J'ai pris comme base pour cette lecture la Cour d'Assises brésilienne (Tribunal de Júri) et plus précisément la Deuxième Cour d'Assises de Belo Horizonte.

9. Expression utilisée par Correa (1983) et adoptée dans ce travail

10. Nous avons préféré garder l'abréviation portugaise BO qui nous paraît plus simple.

11. La structure des questions du BO doit suivre une structure similaire à celle du lead, car comme celui-ci, il doit essayer de répondre à des questions du type : Qui ? (personnages) ; Quoi ? (actes, scénario); Quand? (jour, heure, moment); Où ? (le lieu de l'événement) ; Comment? (la manière dont s'est passée l'événement); Pourquoi ? (la cause de l'événement si elle est connue).

12. Les informations concernant les noms, adresse et autres données relatives à l'identité des personnes citées dans le procès ont été supprimées dans le but de protéger leur intimité.

13. Il s'agit d'une traduction qui essaie de reproduire littéralement la manière d'écrire du policier. Pour une meilleure évaluation de ce texte nous le reproduisons ci-après dans son intégralité en portugais: « Comparecemos ao local do supra, onde fomos informado pela primeira test. que, ouviu uns tiros, aprox. as 01:15 hs no int. da resid. onde trabalha, e logo após, seu patrão, saiu no portão, tomando rumo ignorado, não comparecendo até o presente momento. No local, constatei que, a vitima se encontra, sem vida, estirada no piso, no [ininteligível] do quarto em sua resid. Portanto comuniquei a Copom, enviando os Peritos R e C e O. Passando ao fato 
(ocorrencia) p/ os mesmos. Trasferido a vossa pessoa ac. Citada. A arma do crime não podemos constatar e ferimentos a tiros foram 05 a 06 tiros ».

14. Il faut souligner qu'en Cour d'Assises, ce sont les sept jurés (ou membres du Conseil de Sentence), simples citoyens, honnêtes, qui vont juger les actes de l'accusé, à partir de ce qui leur est présenté par les parties (défense et accusation).

\section{RÉSUMÉS}

Dans cet article tiré d'une étude plus globale sur le fonctionnement du discours véhiculé dans et par la Cour d'Assises au Brésil, notre objectif est d'entamer une réflexion sur le rôle de l'émotion dans la construction argumentative des discours constitutifs de cette instance judiciaire, en tenant compte des relations de pouvoir qu'elle instaure. Pour cela on procède à l'analyse du «Boletim de Ocorrências» (BO), document rédigé par un policier sur les lieux du crime et qui constitue la première mise en scène de l'affaire criminelle.

In this paper, which is part of a wider research about how discourse functions within the criminal court in Brazil, we investigate the role of emotion in the argumentative construction of the discourse of the court, taking into account the power relations of the latter. Thus, we analyze the "Boletim de Ocorrências" or "BO", a police report written on the scene of the crime and which represents the first "stage setting" of the "criminal case".

\section{INDEX}

Mots-clés : argumentation, Cour d'Assises, émotion

Keywords : argumentation, court jury, emotion, Police report

\section{AUTEUR}

\section{HELCIRA MARIA RODRIGUES DE LIMA}

Universidade Federal de Minas Gerais 